\title{
SPECTRAL DECOMPOSITION OF AN ELEMENTARY 3-FERMION 2-BODY OPERATOR
}

\author{
Hubert Grudziński \\ Department of Physics, Academy of Bydgoszcz \\ 85-072 Bydgoszcz, pl. Weyssenhoffa 11, Poland \\ (e-mail: hubertg@ab-byd.edu.pl) \\ Jacek Hirsch \\ Institute of Physics, Nicholas Copernicus University \\ 87-100 Torun, Poland \\ (e-mail: jacekh@phys.uni.torun.pl)
}

\begin{abstract}
The eigenvalues and eigenfunctions of an elementary 3 -fermion 2 -body operator $3 P_{g}^{2} \wedge I^{1} \equiv$ $A^{3} \sum_{1 \leq i<j \leq 3} P_{g}^{2}(i, j) A^{3}$ acting on a 3-particle antisymmetric finite dimensional Hilbert space have been found. Here $P_{g}^{2}$ denotes the projection operator onto a 2-particle antisymmetric function $g^{2}$, while $A^{3}$ denotes the 3 -particle antisymmetrizing operator.
\end{abstract}

keywords: spectral decomposition of operators, fermion 2-body operators

\section{Introduction}

The spectral decomposition of operators is an interesting subject in its own right. This paper arose while we were searching for new conditions for fermion $N$-representability [1]-7], 11, 12. The new condition for fermion $N$-representability, the "dual $P$-condition" [8], requires a knowledge of the maximal eigenvalue of the positive semidefinite operator $\left(\begin{array}{c}N \\ 2\end{array}\right) P_{g}^{2} \wedge I^{\wedge(N-2)}$ acting on an $N$ particle antisymmetric Hilbert space $\mathcal{H}^{\wedge N}$ (the $N$-fold Grassmann product of $\mathcal{H}^{1}$ ), where $P_{g}^{2}$ is the projection operator onto a 2-particle antisymmetric function $g^{2} \in \mathcal{H}^{\wedge 2}$, and $I^{\wedge(N-2)}$ denotes the identity operator on $\mathcal{H}^{\wedge(N-2)}$. We call this operator an elementary $N$-fermion 2-body operator ("with 2-body interactions"). So far, we were able to find the spectral decomposition of such an 
operator for arbitrary $g^{2}$ only for $N=3$, and this paper contains the results. It is realistic to solve the problem for arbitrary $N$ if $g^{2}$ is of a special type, e.g. "extreme geminal" [5], and we will publish those results later. Having the spectral decomposition of an elementary $N$-fermion 2-body operator it is possible to find the reduced 2-particle density operators corresponding to its eigenfunctions and thus obtain some detailed information about the structure of the convex set $\mathcal{P}_{N}^{2}$ consisting of all 2-particle fermion $N$-representable density operators. Especially, the reduced 2-density operators corresponding to the kernel (null-space) of the operator $\left(\begin{array}{c}N \\ 2\end{array}\right) P_{g}^{2} \wedge I^{\wedge(N-2)}$ are interesting because they form a face in the set $\mathcal{P}_{N}^{2}$ exposed by the operator $P_{g}^{2}$ [11]. We have these results for $N=3$ (they will be published in a separate paper), and for this purpose we have introduced in this paper in the null-space of the operator $3 P_{g}^{2} \wedge I^{1}$ an orthonormal basis, and give explicitly the projection operator onto the kernel. Theorem 1 contains the spectral decomposition of the operator $3 P_{g}^{2} \wedge I^{1}$, while Theorem 2 gives the projection operator $\operatorname{Ker}\left(3 P_{g}^{2} \wedge I^{1}\right)$ (upper-case K) onto the null-space $\operatorname{ker}\left(3 P_{g}^{2} \wedge I^{1}\right)$ (lower-case k) of this operator.

\section{Spectral decomposition}

Theorem 1 Let $\mathcal{H}^{1}$ be a finite dimensional Hilbert space $\left(\operatorname{dim} \mathcal{H}^{1}=n\right)$, and $\mathcal{H}^{\wedge 2} \equiv \mathcal{H}^{1} \wedge \mathcal{H}^{1}$ denotes the 2-particle antisymmetric space generated by $\mathcal{H}^{1}$ (the 2-fold Grassmann product of $\mathcal{H}^{1}$ ). Let $P_{g}^{2}$ denote the 1-dimensional projection operator onto a 2-particle antisymmetric function $g^{2} \in \mathcal{H}^{\wedge 2}$ of 1-rank $r=2 s$ possessing the canonical decomposition $g^{2}=\sum_{i=1}^{s} \xi_{i}|2 i-1,2 i\rangle$ with $\sum_{i=1}^{s}\left|\xi_{i}\right|^{2}=1$, where $|2 i-1,2 i\rangle \equiv \sqrt{2} \varphi_{2 i-1}^{1} \wedge \varphi_{2 i}^{1} \equiv \frac{1}{\sqrt{2}} \operatorname{det}\left(\varphi_{2 i-1}^{1}, \varphi_{2 i}^{1}\right), \varphi_{i}^{1} \in \mathcal{H}^{1}$, is the 2-particle normalized determinant. Let the identity operator $I^{1}$ on $\mathcal{H}^{1}$ possess the decomposition $I^{1}=\sum_{i=1}^{r=2 s} P_{i}^{1}+\sum_{i=r+1}^{n} P_{i}^{1}$, where $P_{i}^{1} \equiv \varphi_{i}^{1} \otimes \bar{\varphi}_{i}^{1} \equiv|i\rangle\langle i|(i=1, \ldots, n)$ are 1-dim mutually orthogonal projection operators onto the 1-particle functions $|i\rangle \equiv \varphi_{i}^{1} \in \mathcal{H}^{1}(i=1, \ldots, n)$ forming an orthonormal basis in $\mathcal{H}^{1}$. Then, the 3-particle operator $3 P_{g}^{2} \wedge I^{1}: \mathcal{H}^{\wedge 3} \longrightarrow \mathcal{H}^{\wedge 3}$ possesses the following spectral decomposition

$$
3 P_{g}^{2} \wedge I^{1}=\sum_{k=1}^{s=r / 2}\left(1-\left|\xi_{k}\right|^{2}\right)\left(P_{g_{2 k-1}}^{3}+P_{g_{2 k}}^{3}\right)+\sum_{l=r+1}^{n} P_{g_{l}}^{3}+0 \cdot \operatorname{Ker}\left(3 P_{g}^{2} \wedge I^{1}\right) .
$$

Here, $P_{g_{2 k-1}}^{3}, P_{g_{2 k}}^{3}(k=1, \ldots, s=r / 2), P_{g_{l}}^{3}(l=r+1, \ldots, n)$ are 1-dim projectors onto the following eigenfunctions: 


$$
\begin{gathered}
g_{2 k-1}^{3}=\frac{1}{\sqrt{1-\left|\xi_{k}\right|^{2}}} \sum_{\substack{i=1 \\
(i \neq k)}}^{s} \xi_{i}|2 i-1,2 i, 2 k-1\rangle=\sqrt{\frac{3}{1-\left|\xi_{k}\right|^{2}}} g^{2} \wedge \varphi_{2 k-1}^{1}, \\
\quad\left(k=1, \ldots, s=\frac{r}{2}\right) \\
g_{2 k}^{3}=\frac{1}{\sqrt{1-\left|\xi_{k}\right|^{2}}} \sum_{\substack{i=1 \\
(i \neq k)}}^{s} \xi_{i}|2 i-1,2 i, 2 k\rangle=\sqrt{\frac{3}{1-\left|\xi_{k}\right|^{2}}} g^{2} \wedge \varphi_{2 k}^{1}, \\
g_{l}^{3}=\sum_{i=1}^{s} \xi_{i}|2 i-1,2 i, l\rangle=\sqrt{3} g^{2} \wedge \varphi_{l}^{1}, \quad(l=r+1, \ldots, n),
\end{gathered}
$$

while $\operatorname{Ker}\left(3 P_{g}^{2} \wedge I^{1}\right)$ denotes the projection operator onto the null-space $\operatorname{ker}\left(3 P_{g}^{2} \wedge I^{1}\right)$ of the operator $3 P_{g}^{2} \wedge I^{1}$, which is of dimension $\left(\begin{array}{l}n \\ 3\end{array}\right)-n$. The symbols of the type $|2 i-1,2 i, 2 k-1\rangle \equiv \sqrt{3 !} \varphi_{2 i-1}^{1} \wedge$ $\varphi_{2 i}^{1} \wedge \varphi_{2 k-1}^{1}$ denote the appropriate 3-particle normalized determinants.

Proof. First, we observe that the functions defined by (24) are normalized and all are mutually orthogonal, because the determinants differ from each other in at least one 1-particle function. To prove that they are the eigenfunctions belonging to non-zero eigenvalues of the operator $3 P_{g}^{2} \wedge I^{1}$, we express $P_{g}^{2}$ and $I^{1}$ in the following way:

$$
\begin{aligned}
& P_{g}^{2}=\sum_{i, j=1}^{s=r / 2} \xi_{i} \bar{\xi}_{j}|2 i-1,2 i\rangle\langle 2 j-1,2 j| \\
& I^{1}=\sum_{k=1}^{s=r / 2}\left(P_{2 k-1}^{1}+P_{2 k}^{1}\right)+\sum_{l=r+1}^{n} P_{l}^{1}=\sum_{k=1}^{s}(|2 k-1\rangle\langle 2 k-1|+| 2 k\rangle\langle 2 k|)+\sum_{l=r+1}^{n}|l\rangle\langle l| .
\end{aligned}
$$


Then,

$$
\begin{aligned}
3 P_{g}^{2} \wedge I^{1} & =3 \sum_{i, j=1}^{s} \sum_{k=1}^{s} \xi_{i} \bar{\xi}_{j}|2 i-1,2 i\rangle\langle 2 j-1,2 j| \wedge(|2 k-1\rangle\langle 2 k-1|+| 2 k\rangle\langle 2 k|)+ \\
& +3 \sum_{i, j=1}^{s} \sum_{l=r+1}^{n} \xi_{i} \bar{\xi}_{j}|2 i-1,2 i\rangle\langle 2 j-1,2 j|\wedge| l\rangle\langle l|= \\
& =\sum_{k=1}^{s}\left(1-\left|\xi_{k}\right|^{2}\right)\left(\frac{1}{1-\left|\xi_{k}\right|^{2}} \sum_{\substack{i, j=1 \\
(i, j \neq k)}}^{s} \xi_{i} \bar{\xi}_{j}|2 i-1,2 i, 2 k-1\rangle\langle 2 j-1,2 j, 2 k-1|+\right. \\
& \left.+\frac{1}{1-\left|\xi_{k}\right|^{2}} \sum_{\substack{i, j=1 \\
(i, j \neq k}}^{s} \xi_{i} \bar{\xi}_{j}|2 i-1,2 i, 2 k\rangle\langle 2 j-1,2 j, 2 k|\right)+ \\
& +\sum_{l=r+1}^{n}\left(\sum_{i, j=1}^{s} \xi_{i} \bar{\xi}_{j}|2 i-1,2 i, l\rangle\langle 2 j-1,2 j, l|\right)= \\
& =\sum_{k=1}^{s}\left(1-\left|\xi_{k}\right|^{2}\right)\left(\left|g_{2 k-1}^{3}\right\rangle\left\langle g_{2 k-1}^{3}|+| g_{2 k}^{3}\right\rangle\left\langle g_{2 k}^{3}\right|\right)+\sum_{l=r+1}^{n}\left|g_{l}^{3}\right\rangle\left\langle g_{l}^{3}\right|= \\
& =\sum_{k=1}^{s}\left(1-\left|\xi_{k}\right|^{2}\right)\left(P_{g_{2 k-1}}^{3}+P_{g_{2 k}}^{3}\right)+\sum_{l=r+1}^{n} P_{g_{l}}^{3} \cdot
\end{aligned}
$$

In the proof we have used the fact that $\sqrt{3}|2 i-1,2 i\rangle \wedge|k\rangle=|2 i-1,2 i, k\rangle \equiv \sqrt{3 !} \varphi_{2 i-1}^{1} \wedge \varphi_{2 i}^{1} \wedge$ $\varphi_{k}^{1} \equiv \operatorname{det}\left(\varphi_{2 i-1}^{1}, \varphi_{2 i}^{1}, \varphi_{k}^{1}\right)$. The symbol of the type $\left|g_{2 k-1}^{3}\right\rangle\left\langle g_{2 k-1}^{3}\right| \equiv g_{2 k-1}^{3} \otimes \bar{g}_{2 k-1}^{3} \equiv P_{g_{2 k-1}}^{3}$ denotes the projection operator onto the function $g_{2 k-1}^{3} \in \mathcal{H}^{\wedge 3}$, while $|2 i-1,2 i, k\rangle\langle 2 i-1,2 i, k|$ is the projection operator onto the determinant function $|2 i-1,2 i, k\rangle \in \mathcal{H}^{\wedge 3}$.

Since the 1-dimensional projectors $P_{g_{2 k-1}}^{3}, P_{g_{2 k}}^{3}, P_{g_{l}}^{3}$ are mutually orthogonal, the obtained above expression

$$
3 P_{g}^{2} \wedge I^{1}=\sum_{k=1}^{s}\left(1-\left|\xi_{k}\right|^{2}\right)\left(P_{g_{2 k-1}}^{3}+P_{g_{2 k}}^{3}\right)+\sum_{l=r+1}^{n} P_{g_{l}}^{3}
$$

is the spectral resolution of the operator $3 P_{g}^{2} \wedge I^{1}$ corresponding to the $n$ non-zero eigenvalues. The orthogonal complement in $\mathcal{H}^{\wedge 3}$ of the subspace spanned by the eigenfunctions $g_{2 k-1}^{3}, g_{2 k}^{3}(k=$ $1, \ldots, s=r / 2), g_{l}^{3}(l=r+1, \ldots, n)$ is the null-space (kernel) of the operator $3 P_{g}^{2} \wedge I^{1}$ corresponding to the eigenvalue zero. The projection operator onto this $\left(\begin{array}{l}n \\ 3\end{array}\right)-n$ dimensional null-space we denote by $\operatorname{Ker}\left(3 P_{g}^{2} \wedge I^{1}\right)$. Thus, we have obtained resolution (1), and this completes the proof.

Since we are interested in the reduced density operators corresponding to the eigenfunctions belonging to the eigenvalue zero of the operator $3 P_{g}^{2} \wedge I^{1}$, we have introduced an orthonormal basis in the null-space $\operatorname{ker}\left(3 P_{g}^{2} \wedge I^{1}\right)$ and have found the projection operator $\operatorname{Ker}\left(3 P_{g}^{2} \wedge I^{1}\right)$ onto this null-space explicitly. 
Theorem 2 The projection operator $\operatorname{Ker}\left(3 P_{g}^{2} \wedge I^{1}\right)$ onto the null-space of the operator $3 P_{g}^{2} \wedge I^{1}$ $\left(g^{2} \in \mathcal{H}^{\wedge 2}\right)$ is a sum of mutually orthogonal projectors

$$
\operatorname{Ker}\left(3 P_{g}^{2} \wedge I^{1}\right)=K_{0,3}^{3}+K_{1,2}^{3}+K_{2,1}^{3}+K_{3,0}^{3},
$$

corresponding to the orthogonal decomposition of the 3-particle antisymmetric Hilbert space

$$
\mathcal{H}^{\wedge 3}=\widetilde{\mathcal{R}}^{\wedge 3} \oplus \mathcal{R}^{1} \wedge \widetilde{\mathcal{R}}^{\wedge 2} \oplus \mathcal{R}^{\wedge 2} \wedge \widetilde{\mathcal{R}}^{1} \oplus \mathcal{R}^{\wedge 3}
$$

with $\mathcal{H}^{1}=\mathcal{R}^{1} \oplus \widetilde{\mathcal{R}}^{1}$, where $\mathcal{R}^{1}$ denotes the subspace spanned by the orthonormal basis $\left\{\varphi_{i}^{1}\right\}_{i=1}^{r}$, and $\widetilde{\mathcal{R}}^{1}$ by $\left\{\varphi_{i}^{1}\right\}_{i=r+1}^{n}$. The projection operators $K_{0,3}^{3}, K_{1,2}^{3}, K_{2,1}^{3}, K_{3,0}^{3}$ can be expressed in the following form:

$$
\begin{aligned}
& K_{0,3}^{3}=\sum_{r+1 \leq j_{1}<j_{2}<j_{3} \leq n} P_{j_{1}, j_{2}, j_{3}}^{3}, \\
& K_{1,2}^{3}=\sum_{i=1}^{s} \sum_{r+1 \leq j_{1}<j_{2} \leq n}\left(P_{2 i-1, j_{1}, j_{2}}^{3}+P_{2 i, j_{1}, j_{2}}^{3}\right), \\
& K_{2,1}^{3}=\sum_{1 \leq i_{1}<i_{2} \leq s} \sum_{j=r+1}^{n}\left(P_{2 i_{1}-1,2 i_{2}-1, j}^{3}+P_{2 i_{1}, 2 i_{2}, j}^{3}+P_{2 i_{1}-1,2 i_{2}, j}^{3}+P_{2 i_{1}, 2 i_{2}-1, j}^{3}\right)+ \\
&+\sum_{l=r+1}^{n} \sum_{m=2}^{s} P_{f_{l, m}}^{3}, \\
&=\sum_{1 \leq i_{1}<i_{2}<i_{3} \leq s}\left(P_{2 i_{1}-1,2 i_{2}-1,2 i_{3}-1}^{3}+P_{2 i_{1}-1,2 i_{2}-1,2 i_{3}}^{3}+P_{2 i_{1}-1,2 i_{2}, 2 i_{3}-1}^{3}+\right. \\
&\left.+P_{2 i_{1}, 2 i_{2}-1,2 i_{3}-1}^{3}+P_{2 i_{1}-1,2 i_{2}, 2 i_{3}}^{3}+P_{2 i_{1}, 2 i_{2}-1,2 i_{3}}^{3}+P_{2 i_{1}, 2 i_{2}, 2 i_{3}-1}^{3}+P_{2 i_{1}, 2 i_{2}, 2 i_{3}}^{3}\right)+ \\
& \sum_{k=1}^{s}\left(P_{f_{2 k-1, m}}^{3}+P_{f_{2 k, m}}^{3}\right), \quad \text { for } \quad k=1 \\
&\left\{\begin{array}{l}
\{3, \ldots, s\}, \\
\{2, \ldots, k-1, k+1, \ldots, s\}, \quad \text { for } \quad k=2, \ldots, s .
\end{array}\right.
\end{aligned}
$$

Here, $P_{i j k}^{3}$ denotes a projection operator onto the determinant $|i j k\rangle$, while $P_{f_{l, m}}^{3}, P_{f_{2 k-1, m}}^{3}, P_{f_{2 k, m}}^{3}$, 
are projection operators onto the following functions respectively:

$$
\begin{aligned}
& f_{l, m}^{3}= N_{l m}\left(\sum_{i=1}^{m-1} \xi_{i} \bar{\xi}_{m}|2 i-1,2 i, l\rangle-\sum_{i=1}^{m-1}\left|\xi_{i}\right|^{2}|2 m-1,2 m, l\rangle\right), \\
& N_{l m}=\left(\sum_{i=1}^{m-1}\left|\xi_{i}\right|^{2}\right)^{-\frac{1}{2}}\left(\sum_{i=1}^{m}\left|\xi_{i}\right|^{2}\right)^{-\frac{1}{2}}, \\
& f_{2 k-1, m}^{3}=N_{k m}\left(\sum_{\substack{i=1 \\
i \neq k)}}^{m-1} \xi_{i} \bar{\xi}_{m}|2 i-1,2 i, 2 k-1\rangle-\sum_{\substack{i=1 \\
(i \neq k)}}^{m-1}\left|\xi_{i}\right|^{2}|2 m-1,2 m, 2 k-1\rangle\right), \\
& f_{2 k, m}^{3}=N_{k m}\left(\sum_{\substack{i=1 \\
(i \neq k)}}^{m-1} \xi_{i} \bar{\xi}_{m}|2 i-1,2 i, 2 k\rangle-\sum_{\substack{i=1 \\
(\neq k)}}^{m-1}\left|\xi_{i}\right|^{2}|2 m-1,2 m, 2 k\rangle\right), \\
& N_{k m}=\left(\sum_{\substack{i=1 \\
(i \neq k)}}^{m-1}\left|\xi_{i}\right|^{2}\right)^{-\frac{1}{2}}\left(\sum_{\substack{i=1 \\
(i \neq k)}}^{m}\left|\xi_{i}\right|^{2}\right)^{-\frac{1}{2}} \cdot
\end{aligned}
$$

Proof. The canonical expansion of $g^{2}=\sum_{i=1}^{s=r / 2} \xi_{i} \sqrt{2} \varphi_{2 i-1}^{1} \wedge \varphi_{2 i}^{1} \equiv \sum_{i=1}^{s=r / 2} \xi_{i}|2 i-1,2 i\rangle$ determines the decomposition of $\mathcal{H}^{1}=\mathcal{R}^{1} \oplus \widetilde{\mathcal{R}}^{1}$ and the basis $\left\{\varphi_{i}^{1}\right\}_{i=1}^{n}$ in $\mathcal{H}^{1}$, where the functions $\left\{\varphi_{i}^{1}\right\}_{i=1}^{r}$ span the subspace $\mathcal{R}^{1}$, while $\left\{\varphi_{i}^{1}\right\}_{i=r+1}^{n}$ is an orthonormal basis in the orthogonal complement $\widetilde{\mathcal{R}}^{1}$ of the subspace $\mathcal{R}^{1}$ in $\mathcal{H}^{1}$. Correspondingly, the identity operator $I^{1}$ on $\mathcal{H}^{1}$ has the decomposition $I^{1}=\sum_{i=1}^{r} P_{i}^{1}+\sum_{i=r+1}^{n} P_{i}^{1} \equiv P_{1: r}^{1}+\widetilde{P}_{1: r}^{1}$, which induces the decomposition of the identity operator $I^{\wedge 3}$ on $\mathcal{H}^{\wedge 3}$ onto mutually orthogonal projection operators:

$$
I^{\wedge 3}=\left(P_{1: r}^{1}+\widetilde{P}_{1: r}^{1}\right)^{\wedge 3}=\sum_{k=0}^{3}\left(\begin{array}{l}
3 \\
k
\end{array}\right) P_{1: r}^{\wedge k} \wedge \widetilde{P}_{1: r}^{\wedge(3-k)}=\widetilde{P}_{1: r}^{\wedge 3}+3 P_{1: r}^{1} \wedge \widetilde{P}_{1: r}^{\wedge 2}+3 P_{1: r}^{\wedge 2} \wedge \widetilde{P}_{1: r}^{1}+P_{1: r}^{\wedge 3},
$$

to which in turn corresponds the decomposition of the 3 -particle antisymmetric space $\mathcal{H}^{\wedge 3}$ onto the mutually orthogonal subspaces:

$$
\mathcal{H}^{\wedge 3}=\widetilde{\mathcal{R}}^{\wedge 3} \oplus \mathcal{R}^{1} \wedge \widetilde{\mathcal{R}}^{\wedge 2} \oplus \mathcal{R}^{\wedge 2} \wedge \widetilde{\mathcal{R}}^{1} \oplus \mathcal{R}^{\wedge 3}
$$

(for proof of the above formulae see e.g. [9], 10]). The subspaces on the r.h.s. of (15) are spanned by 3-particle determinants which differ between themselves in the number of 1-particle functions belonging to $\mathcal{R}^{1}$ and $\widetilde{\mathcal{R}}^{1}$ (e.g. $\widetilde{\mathcal{R}}^{\wedge 3}$ is spanned by $\left\{\left|j_{1}, j_{2}, j_{3}\right\rangle\right\}_{j}, \varphi_{j}^{1} \in \widetilde{\mathcal{R}}^{1}$, while $\mathcal{R}^{1} \wedge \widetilde{\mathcal{R}}^{\wedge 2}$ by $\left\{\left|i, j_{1}, j_{2}\right\rangle\right\}_{i, j}, \varphi_{i}^{1} \in \mathcal{R}^{1}, \varphi_{j}^{1} \in \widetilde{\mathcal{R}}^{1}$, etc. $)$.

Now, comparing the spectral decomposition of the operator $3 P_{g}^{2} \wedge I^{1}$ (11) with the decomposition of the identity operator $I^{\wedge 3}$ (14) it is possible to find all the mutually orthogonal projection operators

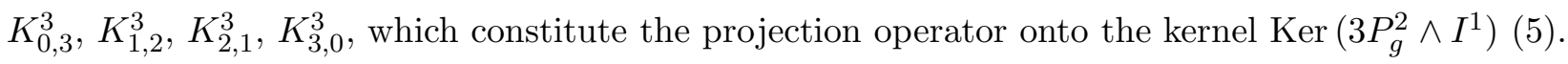


$\left.K_{0,3}^{3}\right) \quad K_{0,3}^{3}$ denotes the projection operator onto the kernel of $3 P_{g}^{2} \wedge I^{1}$ which is contained in the subspace $\widetilde{\mathcal{R}}^{\wedge 3}$ (there are no 1-particle functions from $\mathcal{R}^{1}$ in the 3 -particle determinants). It is seen from (11) that the whole subspace $\widetilde{\mathcal{R}}^{\wedge 3}$ is in the kernel. Hence, from (14):

$$
K_{0,3}^{3}=\widetilde{P}_{1: r}^{\wedge 3}=\sum_{j_{1}, j_{2}, j_{3}=r+1}^{n} P_{j_{1}}^{1} \wedge P_{j_{2}}^{1} \wedge P_{j_{3}}^{1}=\sum_{r+1 \leq j_{1}<j_{2}<j_{3} \leq n} P_{j_{1}, j_{2}, j_{3}}^{3},
$$

where $P_{j}^{1}$ denotes the projection operator onto $\varphi_{j}^{1} \in \widetilde{\mathcal{R}}^{1}$, while $P_{j_{1}, j_{2}, j_{3}}^{3}$ is the projection operator onto the 3 -particle determinant $\left|j_{1}, j_{2}, j_{3}\right\rangle \in \widetilde{\mathcal{R}}^{\wedge 3}$. The dimension of the subspace onto which projects $K_{0,3}^{3}$ : dim range $K_{0,3}^{3}=\left(\begin{array}{c}n-r \\ 3\end{array}\right)=\left(\begin{array}{c}n-2 s \\ 3\end{array}\right)$.

$\left.K_{1,2}^{3}\right)$ There are no eigenfunctions in (11) belonging to the non-zero eigenvalues which are built up from determinants containing only one function $\varphi_{i}^{1} \in \mathcal{R}^{1}$. Therefore,

$$
\begin{aligned}
K_{1,2}^{3} & =3 P_{1: r}^{1} \wedge \widetilde{P}_{1: r}^{\wedge 2}=\sum_{i=1}^{r} \sum_{r+1 \leq j_{1}<j_{2} \leq n} 3 P_{i}^{1} \wedge P_{j_{1}, j_{2}}^{2}=\sum_{i=1}^{r} \sum_{r+1 \leq j_{1}<j_{2} \leq n} P_{i, j_{1}, j_{2}}^{3}= \\
& =\sum_{i=1}^{s=r / 2} \sum_{r+1 \leq j_{1}<j_{2} \leq n}\left(P_{2 i-1, j_{1}, j_{2}}^{3}+P_{2 i, j_{1}, j_{2}}^{3}\right),
\end{aligned}
$$

and dim range $K_{1,2}^{3}=r\left(\begin{array}{c}n-r \\ 2\end{array}\right)=2 s\left(\begin{array}{c}n-2 s \\ 2\end{array}\right)$.

$\left.K_{2,1}^{3}\right)$ First, we decompose the projection operator onto the subspace $\mathcal{R}^{\wedge 2} \wedge \widetilde{\mathcal{R}}^{1}$ in the following way:

$$
\begin{aligned}
3 P_{1: r}^{\wedge 2} & \wedge \widetilde{P}_{1: r}^{1}=3\left[\sum_{i_{1}=1}^{s}\left(P_{2 i_{1}-1}^{1}+P_{2 i_{1}}^{1}\right)\right] \wedge\left[\sum_{i_{2}=1}^{s}\left(P_{2 i_{2}-1}^{1}+P_{2 i_{2}}^{1}\right)\right] \wedge \sum_{j=r+1}^{n} P_{j}^{1}= \\
& =3\left[\sum _ { i _ { 1 } = 1 } ^ { s } \sum _ { i _ { 2 } = 1 } ^ { s } \left(P_{2 i_{1}-1}^{1} \wedge P_{2 i_{2}-1}^{1}+P_{2 i_{1}}^{1} \wedge P_{2 i_{2}}^{1}+P_{2 i_{1}-1}^{1} \wedge P_{2 i_{2}}^{1}+\right.\right. \\
& \left.\left.+P_{2 i_{1}}^{1} \wedge P_{2 i_{2}-1}^{1}\right)\right] \wedge \sum_{j=r+1}^{n} P_{j}^{1}=\sum_{1 \leq i_{1}<i_{2} \leq s} \sum_{j=r+1}^{n}\left(P_{2 i_{1}-1,2 i_{2}-1, j}^{3}+P_{2 i_{1}, 2 i_{2}, j}^{3}+\right. \\
& \left.+P_{2 i_{1}-1,2 i_{2}, j}^{3}+P_{2 i_{1}, 2 i_{2}-1, j}^{3}\right)+\sum_{i=1}^{s} \sum_{j=r+1}^{n} P_{2 i-1,2 i, j}^{3} .
\end{aligned}
$$

Comparing this decomposition with (4) we see that only in the last subspace range $\left(\sum_{i=1}^{s} \sum_{j=r+1}^{n}\right.$ $\left.P_{2 i-1,2 i, j}^{3}\right)$ are there eigenfunctions $g_{l}^{3}(l=r+1, \ldots, n)$ belonging to the eigenvalue different from zero. The dimension of this subspace is $s(n-r)=s(n-2 s)$. There are $n-r$ orthonormal functions $g_{l}^{3}$. Hence, there still exists a $s(n-r)-(n-r)=(s-1)(n-2 s)$ dimensional subspace belonging to the null-space of $3 P_{g}^{2} \wedge I^{1}$. In this subspace we introduce the following 
orthonormal basis:

$$
\begin{aligned}
f_{l, m}^{3}= & N_{l m}\left(\sum_{i=1}^{m-1} \xi_{i} \bar{\xi}_{m}|2 i-1,2 i, l\rangle-\sum_{i=1}^{m-1}\left|\xi_{i}\right|^{2}|2 m-1,2 m, l\rangle\right) \\
& N_{l m}=\left(\sum_{i=1}^{m-1}\left|\xi_{i}\right|^{2}\right)^{-\frac{1}{2}}\left(\sum_{i=1}^{m}\left|\xi_{i}\right|^{2}\right)^{-\frac{1}{2}}, \quad l=r+1, \ldots, n, \quad m=2, \ldots, s .
\end{aligned}
$$

It can be checked that the functions $\left\{f_{l, m}^{3}\right\}(l=r+1, \ldots, n ; \quad m=2, \ldots, s$.$) are:$

$1^{\circ}$ orthogonal to $\left\{g_{l}^{3}\right\}(l=r+1, \ldots, n)$,

$$
\begin{aligned}
\left\langle g_{l}^{3} \mid f_{l, m}^{3}\right\rangle & =\sum_{i=1}^{s} \bar{\xi}_{i}\langle 2 i-1,2 i, l| N_{l m}\left(\sum_{j=1}^{m-1} \xi_{j} \bar{\xi}_{m}|2 j-1,2 j, l\rangle+\right. \\
& \left.-\sum_{j=1}^{m-1}\left|\xi_{j}\right|^{2}|2 m-1,2 m, l\rangle\right)= \\
& =N_{l m}\left(\sum_{i=1}^{m-1}\left|\xi_{i}\right|^{2} \bar{\xi}_{m}-\sum_{i=1}^{m-1}\left|\xi_{i}\right|^{2} \bar{\xi}_{m}\right)=0,
\end{aligned}
$$

obviously $\left\langle g_{l_{1}}^{3} \mid f_{l_{2}, m}^{3}\right\rangle=0$ if $l_{1} \neq l_{2}$

$2^{\circ}$ normalized,

$$
\begin{aligned}
& \left\langle f_{l, m}^{3} \mid f_{l, m}^{3}\right\rangle=N_{l m}^{2}\left(\sum_{i=1}^{m-1} \bar{\xi}_{i} \xi_{m}\left\langle 2 i-1,2 i,\left.l\left|-\sum_{i=1}^{m-1}\right| \xi_{i}\right|^{2}\langle 2 m-1,2 m, l|\right)\right. \\
& \left(\sum_{j=1}^{m-1} \xi_{j} \bar{\xi}_{m}|2 j-1,2 j, l\rangle-\sum_{j=1}^{m-1}\left|\xi_{j}\right|^{2}|2 m-1,2 m, l\rangle\right)= \\
& =N_{l m}^{2}\left(\sum_{i=1}^{m-1}\left|\xi_{i}\right|^{2}\left|\xi_{m}\right|^{2}+\sum_{i=1}^{m-1}\left|\xi_{i}\right|^{2} \sum_{j=1}^{m-1}\left|\xi_{j}\right|^{2}\right)= \\
& =N_{l m}^{2}\left(\sum_{i=1}^{m-1}\left|\xi_{i}\right|^{2}\right)\left(\left|\xi_{m}\right|^{2}+\sum_{j=1}^{m-1}\left|\xi_{j}\right|^{2}\right)=N_{l m}^{2}\left(\sum_{i=1}^{m-1}\left|\xi_{i}\right|^{2}\right)\left(\sum_{j=1}^{m}\left|\xi_{j}\right|^{2}\right)=1
\end{aligned}
$$


$3^{\circ}$ mutually orthogonal,

$$
\begin{aligned}
& \left\langle f_{l, m_{1}}^{3} \mid f_{l, m_{2}}^{3}\right\rangle_{m_{1}<m_{2}}=N_{l m_{1}} N_{l m_{2}}\left(\sum_{i=1}^{m_{1}-1} \bar{\xi}_{i} \xi_{m_{1}}\left\langle 2 i-1,2 i,\left.l\left|-\sum_{i=1}^{m_{1}-1}\right| \xi_{i}\right|^{2}\left\langle 2 m_{1}-1,2 m_{1}, l\right|\right)\right. \\
& \left(\sum_{j=1}^{m_{2}-1} \xi_{j} \bar{\xi}_{m_{2}}|2 j-1,2 j, l\rangle-\sum_{j=1}^{m_{2}-1}\left|\xi_{j}\right|^{2}\left|2 m_{2}-1,2 m_{2}, l\right\rangle\right)= \\
& =N_{l m_{1}} N_{l m_{2}}\left(\sum_{i=1}^{m_{1}-1} \bar{\xi}_{i} \xi_{m_{1}}\left\langle 2 i-1,2 i,\left.l\left|-\sum_{i=1}^{m_{1}-1}\right| \xi_{i}\right|^{2}\left\langle 2 m_{1}-1,2 m_{1}, l\right|\right)\right. \\
& \left(\sum_{j=1}^{m_{1}-1} \xi_{j} \bar{\xi}_{m_{2}}|2 j-1,2 j, l\rangle+\sum_{j=m_{1}}^{m_{2}-1} \xi_{j} \bar{\xi}_{m_{2}}|2 j-1,2 j, l\rangle-\sum_{j=1}^{m_{2}-1}\left|\xi_{j}\right|^{2}\left|2 m_{2}-1,2 m_{2}, l\right\rangle\right)= \\
& =N_{l m_{1}} N_{l m_{2}}\left(\sum_{i=1}^{m_{1}-1}\left|\xi_{i}\right|^{2} \xi_{m_{1}} \bar{\xi}_{m_{2}}-\sum_{i=1}^{m_{1}-1}\left|\xi_{i}\right|^{2} \xi_{m_{1}} \bar{\xi}_{m_{2}}\right)=0,
\end{aligned}
$$

obviously $\left\langle f_{l_{1}, m}^{3} \mid f_{l_{2}, m}^{3}\right\rangle_{l_{1} \neq l_{2}}=0$.

Thus, the sets $\left\{f_{l, m}^{3}\right\}(l=r+1, \ldots, n ; \quad m=2, \ldots, s)$ and $\left\{g_{l}^{3}\right\}(l=r+1, \ldots, n)$ span the whole subspace range $\left(\sum_{i=1}^{s} \sum_{j=r+1}^{n} P_{2 i-1,2 i, j}^{3}\right)$. Now we can find the dimension of $\operatorname{ker}\left(3 P_{g}^{2} \wedge I^{1}\right)$ contained in $\mathcal{R}^{\wedge 2} \wedge \widetilde{\mathcal{R}}^{1}$. It is equal to dim range $K_{2,1}^{3}=4(n-r)\left(\begin{array}{l}s \\ 2\end{array}\right)+[s(n-r)-(n-r)]=$ $4(n-2 s)\left(\begin{array}{l}s \\ 2\end{array}\right)+(s-1)(n-2 s)$.

$\left.K_{3,0}^{3}\right) \quad K_{3,0}^{3}$ is a projection operator onto the $\operatorname{ker}\left(3 P_{g}^{2} \wedge I^{1}\right)$ which is contained in the subspace $\mathcal{R}^{\wedge 3}$. The dimension of the subspace $\mathcal{R}^{\wedge 3}$ is $\left(\begin{array}{c}r \\ 3\end{array}\right)=\left(\begin{array}{c}2 s \\ 3\end{array}\right)$, and there are $r=2 s$ orthonormal functions $\left\{g_{2 k-1}^{3}, g_{2 k}^{3}\right\}(k=1, \ldots, s)$ belonging to the range $\left(3 P_{g}^{2} \wedge I^{1}\right)$ in $\mathcal{R}^{\wedge 3}$. Hence, the dimension of the null-space in $\mathcal{R}^{\wedge 3}$ is equal to $\left(\begin{array}{c}2 s \\ 3\end{array}\right)-2 s$. To find the projection operator $K_{3,0}^{3}$ onto this nullspace, we have to decompose properly the projection operator $P_{1: r}^{\wedge 3}$ onto the whole subspace $\mathcal{R}^{\wedge 3}$. Here the calculations are longer than in the previous cases considered above, so we give only the milestones.

$$
\begin{aligned}
P_{1: r}^{\wedge 3} & =\sum_{i_{1}, i_{2}, i_{3}=1}^{s}\left(P_{2 i_{1}-1}^{1}+P_{2 i_{1}}^{1}\right) \wedge\left(P_{2 i_{2}-1}^{1}+P_{2 i_{2}}^{1}\right) \wedge\left(P_{2 i_{3}-1}^{1}+P_{2 i_{3}}^{1}\right)= \\
& =\sum_{1 \leq i_{1}<i_{2}<i_{3} \leq s}\left(P_{2 i_{1}-1,2 i_{2}-1,2 i_{3}-1}^{3}+P_{2 i_{1}-1,2 i_{2}-1,2 i_{3}}^{3}+P_{2 i_{1}-1,2 i_{2}, 2 i_{3}-1}^{3}+\right. \\
& \left.+P_{2 i_{1}, 2 i_{2}-1,2 i_{3}-1}^{3}+P_{2 i_{1}-1,2 i_{2}, 2 i_{3}}^{3}+P_{2 i_{1}, 2 i_{2}-1,2 i_{3}}^{3}+P_{2 i_{1}, 2 i_{2}, 2 i_{3}-1}^{3}+P_{2 i_{1}, 2 i_{2}, 2 i_{3}}^{3}\right)+ \\
& +\sum_{\substack{i_{1}, i_{2}=1 \\
\left(i_{1} \neq i_{2}\right)}}^{s}\left(P_{2 i_{1}-1,2 i_{1}, 2 i_{2}-1}^{3}+P_{2 i_{1}-1,2 i_{1}, 2 i_{2}}^{3}\right) .
\end{aligned}
$$

Here we have used the facts that: 


$$
\begin{array}{r}
\sum_{i_{1}, i_{2}, i_{3}=1}^{s} P_{2 i_{1}-1}^{1} \wedge P_{2 i_{2}-1}^{1} \wedge P_{2 i_{3}-1}^{1}=\sum_{1 \leq i_{1}<i_{2}<i_{3} \leq s} 3 ! P_{2 i_{1}-1}^{1} \wedge P_{2 i_{2}-1}^{1} \wedge P_{2 i_{3}-1}^{1}= \\
=\sum_{1 \leq i_{1}<i_{2}<i_{3} \leq s} P_{2 i_{1}-1,2 i_{2}-1,2 i_{3}-1}^{3}
\end{array}
$$

and similarly for the projector with three even indices;

$2^{\circ}$

$$
\begin{aligned}
& \sum_{i_{1}, i_{2}, i_{3}=1}^{s}\left(P_{2 i_{1}-1}^{1} \wedge P_{2 i_{2}-1}^{1} \wedge P_{2 i_{3}}^{1}+P_{2 i_{1}-1}^{1} \wedge P_{2 i_{2}}^{1} \wedge P_{2 i_{3}-1}^{1}+P_{2 i_{1}}^{1} \wedge P_{2 i_{2}-1}^{1} \wedge P_{2 i_{3}-1}^{1}\right)= \\
& \quad=\sum_{i_{1}, i_{2}=1}^{s} P_{2 i_{1}-1}^{1} \wedge P_{2 i_{2}-1}^{1} \wedge P_{2 i_{1}}^{1}+\sum_{i_{1}, i_{2}=1}^{s} P_{2 i_{1}-1}^{1} \wedge P_{2 i_{2}-1}^{1} \wedge P_{2 i_{2}}^{1}+ \\
& \quad+\sum_{\substack{i_{1}, i_{2}, i_{3}=1 \\
\left(i_{1} \neq i_{2} \neq i_{3}\right)}}^{s} P_{2 i_{1}-1}^{1} \wedge P_{2 i_{2}-1}^{1} \wedge P_{2 i_{3}}^{1}+\sum_{i_{1}, i_{3}=1}^{s} P_{2 i_{1}-1}^{1} \wedge P_{2 i_{1}}^{1} \wedge P_{2 i_{3}-1}^{1}+ \\
& \quad+\sum_{\substack{i_{1}, i_{2}=1 \\
i_{2}=1}}^{s} P_{2 i_{1}-1}^{1} \wedge P_{2 i_{2}}^{1} \wedge P_{2 i_{2}-1}^{1}+\sum_{\substack{i_{1}, i_{2}, i_{3}=1 \\
\left(i_{1} \neq i_{2} \neq i_{3}\right)}}^{s} P_{2 i_{1}-1}^{1} \wedge P_{2 i_{2}}^{1} \wedge P_{2 i_{3}-1}^{1}+ \\
& \quad+\sum_{\substack{i_{1}, i_{3}=1 \\
s}}^{s} P_{2 i_{1}}^{1} \wedge P_{2 i_{1}-1}^{1} \wedge P_{2 i_{3}-1}^{1}+\sum_{\substack{i_{1}, i_{2}=1 \\
i_{2}=1}}^{s} P_{2 i_{1}}^{1} \wedge P_{2 i_{2}-1}^{1} \wedge P_{2 i_{1}-1}^{1}+ \\
& \quad+\sum_{\substack{i_{1}, i_{2}, i_{3}=1 \\
\left(i_{1} \neq i_{2} \neq i_{3}\right)}}^{s} P_{2 i_{1}}^{1} \wedge P_{2 i_{2}-1}^{1} \wedge P_{2 i_{3}-1}^{1}=\sum_{\substack{i_{1}, i_{2}=1 \\
\left(i_{1} \neq i_{2}\right)}}^{s} 3 ! P_{2 i_{1}-1}^{1} \wedge P_{2 i_{1}}^{1} \wedge P_{2 i_{2}-1}^{1}+ \\
& \quad+\sum_{\substack{1 \leq i_{1}<i_{2}<i_{3} \leq s \\
s}} 3 !\left(P_{2 i_{1}-1}^{1} \wedge P_{2 i_{2}-1}^{1} \wedge P_{2 i_{3}}^{1}+P_{2 i_{1}-1}^{1} \wedge P_{2 i_{2}}^{1} \wedge P_{2 i_{3}-1}^{1}+P_{2 i_{1}}^{1} \wedge P_{2 i_{2}-1}^{1} \wedge P_{2 i_{3}-1}^{1}\right)= \\
& \quad=\sum_{\substack{i_{1}, i_{2}=1 \\
i_{1} \neq i_{2}}} P_{2 i_{1}-1,2 i_{1}, 2 i_{2}-1}^{3}+\sum_{1 \leq i_{1}<i_{3} \leq s}\left(P_{2 i_{1}-1,2 i_{2}-1,2 i_{3}}^{3}+P_{2 i_{1}-1,2 i_{2}, 2 i_{3}-1}^{3}+P_{2 i_{1}, 2 i_{2}-1,2 i_{3}-1}^{3}\right) .
\end{aligned}
$$

Similarly we proceed with the projectors containing two even and one odd indices.

It follows from (11) that only in the subspace range $\sum_{\substack{i_{1}, i_{2}=1 \\\left(i_{1} \neq i_{2}\right)}}^{s}\left(P_{2 i_{1}-1,2 i_{1}, 2 i_{2}-1}^{3}+P_{2 i_{1}-1,2 i_{1}, 2 i_{2}}^{3}\right)$ are there $r=2 s$ eigenfunctions $\left\{g_{2 k-1}^{3}, g_{2 k}^{3}\right\}(k=1, \ldots, s=r / 2)$ belonging to non-zero eigenvalues of $3 P_{g}^{2} \wedge I^{1}$. Since this subspace is $2 s(s-1)$ dimensional, there still exists in it the $2 s(s-1)-2 s=$ $2 s(s-2)$ dimensional null-space of $3 P_{g}^{2} \wedge I^{1}$. Within this subspace we can establish the following 
orthonormal basis:

$$
\begin{aligned}
f_{2 k-1, m}^{3}= & N_{k m}\left(\sum_{\substack{i=1 \\
i \neq k)}}^{m-1} \xi_{i} \bar{\xi}_{m}|2 i-1,2 i, 2 k-1\rangle-\sum_{\substack{i=1 \\
(i \neq k)}}^{m-1}\left|\xi_{i}\right|^{2}|2 m-1,2 m, 2 k-1\rangle\right), \\
f_{2 k, m}^{3}= & N_{k m}\left(\sum_{\substack{i=1 \\
(i \neq k)}}^{m-1} \xi_{i} \bar{\xi}_{m}|2 i-1,2 i, 2 k\rangle-\sum_{\substack{i=1 \\
(i \neq k)}}^{m-1}\left|\xi_{i}\right|^{2}|2 m-1,2 m, 2 k\rangle\right), \\
N_{k m}= & \left(\sum_{\substack{i=1 \\
(i \neq k)}}^{m-1}\left|\xi_{i}\right|^{2}\right)^{-\frac{1}{2}}\left(\sum_{\substack{i=1 \\
(i \neq k)}}^{m}\left|\xi_{i}\right|^{2}\right)^{-\frac{1}{2}}, \quad \text { for } k=1 \\
& k=1, \ldots, s ; \quad m \in J, \quad J=\left\{\begin{array}{l}
\{3, \ldots, s\}, \\
\{2, \ldots, k-1, k+1, \ldots, s\}, \text { for } k=2, \ldots, s .
\end{array}\right.
\end{aligned}
$$

The orthogonality between any function with "odd index $k$ " to any one with "even index $k$ " as well as between functions with different indices $k$ is seen by inspection. The normalization, and other orthogonality relations can be proved in a way similar to that used in the previous case $K_{2,1}^{3}$, and we omit these calculations for brevity. Now, we can calculate the dimension of the $\operatorname{ker}\left(3 P_{g}^{2} \wedge I^{1}\right)$ contained in $\mathcal{R}^{\wedge 3}$ : it is equal to dim range $K_{3,0}^{3}=8\left(\begin{array}{l}s \\ 3\end{array}\right)+2 s(s-2)$. Thus, in principle the proof is done. To make sure that everything that belongs to the $\operatorname{ker}\left(3 P_{g}^{2} \wedge I^{1}\right)$ is taken into account, we can perform the dimensionality test. The subspace $\mathcal{H}^{\wedge 3}$ is $\left(\begin{array}{l}n \\ 3\end{array}\right)$ dimensional, there are $n$ orthonormal eigenfunctions belonging to the non-zero eigenvalues of the operator $3 P_{g}^{2} \wedge I^{1}$, therefore the dimension of $\operatorname{ker}\left(3 P_{g}^{2} \wedge I^{1}\right)$ is $\left(\begin{array}{l}n \\ 3\end{array}\right)-n$. On the other hand this should be equal to

$$
\begin{aligned}
& \sum_{i=0}^{3} \operatorname{dim} \operatorname{range} K_{i, 3-i}^{3}= \\
& \quad=\left(\begin{array}{c}
n-2 s \\
3
\end{array}\right)+2 s\left(\begin{array}{c}
n-2 s \\
2
\end{array}\right)+\left[4(n-2 s)\left(\begin{array}{l}
s \\
2
\end{array}\right)+(s-1)(n-2 s)\right]+\left[8\left(\begin{array}{l}
s \\
3
\end{array}\right)+2 s(s-2)\right] .
\end{aligned}
$$

This sum is really equal to $\left(\begin{array}{l}n \\ 3\end{array}\right)-n$, and this completes the proof.

\section{References}

[1] A. J. Coleman: Rev. Mod. Phys. 35 (1963), 668.

[2] A. J. Coleman: J. Math. Phys. 13 (1972), 214.

[3] A. J. Coleman: Rep. Math. Phys. 4 (1973), 113.

[4] A. J. Coleman and V. I. Yukalov: Reduced Density Matrices, Springer 2000. 
[5] A. J. Coleman: J. Math. Phys. 6 (1965), 1425.

[6] R. M. Erdahl and V. H. Smith, Jr. (Eds): Density Matrices and Density Functionals (Proceedings of A. J. Coleman Symposium), D. Reidel Publishing Co., Dordrecht 1987.

[7] C. Garrod and J. K. Percus: J. Math. Phys. 5 (1964), 1756.

[8] H. Grudziński and J. Hirsch: Rep. Math. Phys. (in press).

[9] H. Grudziński: Int. J. Quant. Chem. 27 (1985), 709.

[10] H. Grudziński: ref. [6] p.89.

[11] H. Kummer: J. Math. Phys. 8 (1967), 2063.

[12] H. Kummer: Int. J. Quant. Chem. 12 (1977), 1033. 